\title{
Nonlinear Behaviors of Tail Dependence and Cross-Correlation of Financial Time Series Model
}

\author{
Wei Deng and Jun Wang \\ Institute of Financial Mathematics and Financial Engineering, School of Science, Beijing Jiaotong University, Beijing 100044, China
}

Correspondence should be addressed to Jun Wang; wangjun@center.njtu.edu.cn

Received 20 March 2014; Revised 30 April 2014; Accepted 2 May 2014; Published 28 May 2014

Academic Editor: Rehana Naz

Copyright (C) 2014 W. Deng and J. Wang. This is an open access article distributed under the Creative Commons Attribution License, which permits unrestricted use, distribution, and reproduction in any medium, provided the original work is properly cited.

\begin{abstract}
Nonlinear behaviors of tail dependence and cross-correlation of financial time series are reproduced and investigated by stochastic voter dynamic system. The voter process is a continuous-time Markov process and is one of the interacting dynamic systems. The tail dependence of return time series for pairs of Chinese stock markets and the proposed financial models is studied by copula analysis, in an attempt to detect and illustrate the existence of relevant correlation relationships. Further, the multifractality of cross-correlations for return series is studied by multifractal detrended cross-correlation analysis, which indicates the analogous cross-correlations and some fractal characters for both actual data and simulative data and provides an intuitive evidence for market inefficiency.
\end{abstract}

\section{Introduction}

Nonlinear analysis of return time series is an active topic to understand and model distributions of financial price fluctuations, which has long been a focus of economic research. Fluctuations of two related financial markets usually exhibit the correlated (or "indirect" correlated) phenomena, and the approach of analyzing the correlations is definitely significant to realize the inner relations between the financial markets.

There have been considerable results about non-Gaussian characteristics in the fields of economics and finance $[1,2]$. The empirical research shows that there exist fat-tail distributions for financial time series instead of understanding the possibility of large event by employing the Gaussian distribution. The features of peak and fat-tail behaviors of returns are useful to achieve accurate and rational descriptions about stock's volatilities in financial markets, and the corresponding phenomenon is often called fat-tailed Lévy-distribution [13]. In the present work, the approach of studying nonlinear dependence of tails for two return time series has been performed by the copula analysis. With the interpretation of Kendall's $\kappa$, Spearman order correlation coefficient $\rho_{s}$, and tail dependence coefficients $\psi_{u}$ and $\psi_{l}$, we investigate the dependence behaviors of two return series with the copula functions. Copula analysis was introduced for understanding the relationships between multidimensional probability function and its lower dimension margins; see Sklar [4] and Nelsen [5]. Then copula analysis is now widely used in statistics, since copula is the function that connects joint distribution function and marginal distribution functions.

Much progress has been made in the exploration of fractal properties for financial time series. Hurst [6] proposed R/S method to analyze the long memory properties in the hydrological field, deriving the long memory of the time series from tide data. Peng et al. [7] introduced the detrended fluctuation analysis (DFA) to study the characters of DNA sequences, which is effective in the interpretation on the quality of monofractal scaling. In order to indicate the multifractality properties in time sequences, a method called the multifractal detrended fluctuation analysis (MF-DFA) was put forward by Castro E Silva and Moreira [8] and then further developed by Weber and Talkner [9] and Kantelhardt et al. [10]. The MF-DFA method was used in capturing the nature of equity price in financial market and relevant research field $[1,10,11]$. Furthermore, the detrended cross-correlation analysis (DCCA) was developed by Podobnik and Stanley [12] to investigate the power-law cross-correlation between two simultaneously recorded time series in the presence of 
nonstationary behavior, where the cross-correlation between two time series describes the normalized cross covariance function. Zhou [13] proposed a new method which is called multifractal detrended cross-correlation analysis (MF-XDFA) with the combination of MF-DFA and DCCA methods, illustrating the validity in financial time series. Jiang and Zhou [14] put forward MF-X-DMA method which is based on MF-DMA [15] and DMA [16], comparing the performances among the forward, centered, and backward MF-XDMA algorithm. Another extension of multifractal analysis method like MF-XHA [17] is proposed by Kristoufek. In this work, we will make an empirical research at testifying and quantifying cross-correlations between the financial time series by multifractal detrended analysis.

Recently, there has been a considerable interest in the application of statistical physics systems (or interacting particle systems) to financial market dynamics [18-28], since financial modeling is vital in financial time series analysis. The modeling of dynamics of forwards prices is becoming a key problem in financial decision making, such as risk management, physical assets valuation, and derivatives pricing. Any modeling, an abstract representation of reality, aiming at understanding price fluctuations, needs to define a mechanism for the formation of the price, in an attempt to reproduce and explain performance and nature of financial assets. In the present paper, a financial agent-based price model is established by stochastic voter process $[21,22,25,29$, 30 ], and main stylized facts of financial time series of stochastic voter model such as the distribution of returns (power-law distribution for absolute returns), volatility clustering, long memory (long-range correlation in the volatility), and related DFA analysis have been studied [21, 22, 31]. Supported by these outcomes above, we make a further attempt to reveal the empirical laws in correlations of returns based on the voter model as well. The tail dependence and cross-correlation of two return series of the proposed model are studied by copula analysis and MF-DCCA analysis, respectively. Further, the price data of Shanghai Stock Exchange (SSE) Composite Index and Shenzhen Stock Exchange (SZSE) Component Index are selected for the empirical research in order to study the fluctuation correlations of actual market and proposed model by comparison.

\section{Financial Time Series Model}

2.1. Stochastic Voter Dynamic System. Stochastic voter process is a continuous-time Markov process [32] and is one of interacting particle systems (or statistical physics systems) $[29,30,33,34]$. Individuals placed at the points of $\mathbb{Z}^{d}$ might have one of two possible opinions on a political issue (in favor or against); at independent exponential times, an individual reassesses his view by choosing a neighbor at random with certain probabilities and then adopting his position. To write this as a set-valued process, we let $\xi_{\tau}$ be the set of voters in favor. Then the dynamics of the voter model can be formulated as follows. Let $\xi_{\tau}^{A}$ denote the state at time $\tau$ with the initial state set $\xi_{0}^{A}=A$, and let $\xi_{\tau}^{\{0\}}(x)$ be the state of $x \in$ $\mathbb{Z}^{d}$ at time $\tau$ for $\xi_{0}^{\{0\}}=\{0\}$. The stochastic dynamics of voter model $\xi_{\tau}$ is Markov process on a configuration space $\{0,1\}^{\mathbb{Z}^{d}}$ whose generator has the form

$$
\mathscr{A} g(\xi)=\sum_{x \in \mathbb{Z}^{d}} c(x, \xi)\left[g\left(\xi^{x}\right)-g(\xi)\right]
$$

where the functions $g$ on $\{0,1\}^{\mathbb{Z}^{d}}$ depend on finitely many coordinates, and $\xi^{x}(z)=\xi(z)$ if $z \neq x, \xi^{x}(z)=1-\xi(x)$ if $z=x$, for $x, z \in \mathbb{Z}^{d} . c(x, \xi)$ is the transition rate function for the process which is given as follows. For any $\xi \in\{0,1\} \mathbb{Z}^{d}$, the state of $x \in \mathbb{Z}^{d}$ flips according to the transition rates

$$
\begin{aligned}
& 0 \longrightarrow 1 \quad \text { at rate } \lambda \sum_{y \in \mathbb{Z}^{d}} p(x, y) I_{\{\xi(y)=1\}}, \\
& 1 \longrightarrow 0 \quad \text { at rate } \sum_{y \in \mathbb{Z}^{d}} p(x, y) I_{\{\xi(y)=0\}},
\end{aligned}
$$

where $I$ is the indicative function, $p(x, y) \geqslant 0$ for $x, y \in \mathbb{Z}^{d}$, and $\sum_{y \in \mathbb{Z}^{d}} p(x, y)=1$ for all $x \in \mathbb{Z}^{d}$. Here, we suppose that the transition probability $p(x, y)$ is translation invariant and symmetric, such that the Markov chain with those transition probabilities is irreducible. $\lambda$ is an intensity which is called the "carcinogenic advantage" in the voter model. The model is called the voter model for $\lambda=1$, and the model is called the biased voter model for $\lambda>1$. For the biased voter model, there exists a "critical value" for the process which is defined as $[29,30]$

$$
\lambda_{c}=\inf \left\{\lambda: P\left(\left|\xi_{\tau}^{\{0\}}\right|>0, \forall \tau \geqslant 0\right)>0\right\},
$$

where $\left|\xi_{\tau}^{\{0\}}\right|$ is the cardinality of $\xi_{\tau}^{\{0\}}$. Assume $\lambda>\lambda_{c}$; then there is a convex set $C$ so that, on $\Omega_{\infty}=\left\{\xi_{\tau}^{\{0\}} \neq \emptyset, \forall \tau\right\}$, we have for any $\varepsilon>0$ and for all $\tau$ sufficiently large

$$
(1-\varepsilon) \tau C \cap \mathbb{Z}^{d} \subset \xi_{\tau}^{\{0\}} \subset(1+\varepsilon) \tau C \cap \mathbb{Z}^{d} .
$$

If $\lambda<\lambda_{c}$, for some positive $\eta(\lambda)$, we have

$$
P\left(\xi_{\tau}^{\{0\}} \neq \emptyset\right) \leqslant e^{-\eta \tau} .
$$

The above results imply that, on $d$-dimensional lattice, the process becomes vacant exponentially for $\lambda<\lambda_{c}$; the process survives with the positive probability for $\lambda>\lambda_{c}$.

2.2. Financial Agent-Based Price Process. In this section, a financial agent-based price model is developed by the voter interacting system. We suppose that the information about the financial market leads to the volatilities of stock prices. There are three kinds of information including buying information, selling information, and neutral information which classify the investors into their corresponding groups. Suppose each trader can trade the stock several times at each day $t \in\{1,2, \ldots, n\}$, but at most unit number of the stock at each time. Let $l$ be the time length of trading day; we denote the stock price at time $\tau$ in the $t$ th trading day by $P_{t}(\tau)$, where $\tau \in[0, l]$. Assume that the stock is made up of $m+1$ ( $m$ is sufficiently large) investors, who are located 
in a line $\{-m / 2, \ldots,-1,0,1, \ldots, m / 2\} \subset \mathbb{Z}$ (similarly for $d$ dimensional lattice $\mathbb{Z}^{d}$ ) [30]. At the beginning of trading in each day, suppose that only the investor at the origin site "0" receives some information. We define a random variable $\zeta_{t}$ with the values $1,-1$, and 0 to represent this investor holds buying opinion, selling opinion, or neutral opinion with probabilities $p_{1}, p_{-1}$, or $1-p_{1}-p_{-1}[21,22,25]$, respectively. Then, this investor sends bullish, bearish, or neutral signal to his nearest neighbors. According to $d$-dimensional voter process system, investors can affect each other or the information can be transmitted, which is supposed to be the main factor of price fluctuations for the market; see the following in detail. (a) When $\zeta_{t}=1$, if $\xi_{\tau}^{\{0\}}(w)=1$, we say that the investor at $w$ holds buying opinion at time $\tau$, and this investor recovers to neutral position at a rate equal to the number of the vacant neighbors; if $\xi_{\tau}^{\{0\}}(w)=0$, we think the investor at $w$ holds neutral opinion at $\tau$, and this investor is changed to hold buying opinion by his nearest neighbors at rate $\lambda \sum_{v:|w-v|=1} \xi_{\tau}^{\{0\}}(v)$. (b) When $\zeta_{t}=-1$, if $\xi_{\tau}^{\{0\}}(w)=1$, we say that the investor at $w$ holds selling opinion at time $\tau$; also this investor recovers to neutral opinion at a rate equal to the number of the vacant neighbors; if $\xi_{\tau}^{\{0\}}(w)=0$, the investor is changed to hold selling opinion by his nearest neighbors at rate $\lambda \sum_{v:|w-v|=1} \xi_{\tau}^{\{0\}}(v)$. (c) When the initial random variable $\zeta_{t}=0$, the process $\xi_{\tau}^{\{0\}}(w)$ is ignored; this means that the behaviors of investors do not affect the fluctuations of stock prices. For a fixed trading day $t \in\{1, \ldots, n\}$ and $\tau \in[0, l]$, let

$$
M_{t}(\tau)=\frac{\zeta_{t} \cdot\left|\xi_{\tau}^{\{0\}}\right|}{m}
$$

where $\left|\xi_{\tau}^{\{0\}}\right|=\sum_{w=-m / 2}^{m / 2} \xi_{\tau}^{\{0\}}(w)$ and $m$ may depend on the trading days $n$. Then, we define the formula of a discrete time stock price as follows [35]:

$$
P_{t}(\tau)=\exp \left\{\alpha M_{t}(\tau)\right\} P_{t-1}(\tau),
$$

where $P_{0}$ is the stock price at time 0 . According to the theory of the voter process, if $\lambda>\lambda_{c}$, the information will be spread widely, so this will affect the investors positions and at last will affect the fluctuation of the stock price. On the contrary, if $\lambda<\lambda_{c}$, the influence on the stock price by the investors is limited. Further, we give the formula of the stock logarithmic return from time $t-1$ to $t$ as follows:

$$
r(t)=\ln P_{t}(\tau)-\ln P_{t-1}(\tau) .
$$

From the above proposed financial model and the computer simulation, Figure 1 presents the fluctuations of price time series and the corresponding returns, respectively, when intensity $\lambda=3$.

\section{Dependence Behavior of Returns by Copula Analysis}

3.1. Brief Description of Copula Function. Copula theory was put forward by Sklar [4]; copulas provide a convenient way to express joint distributions of two or more random variables.
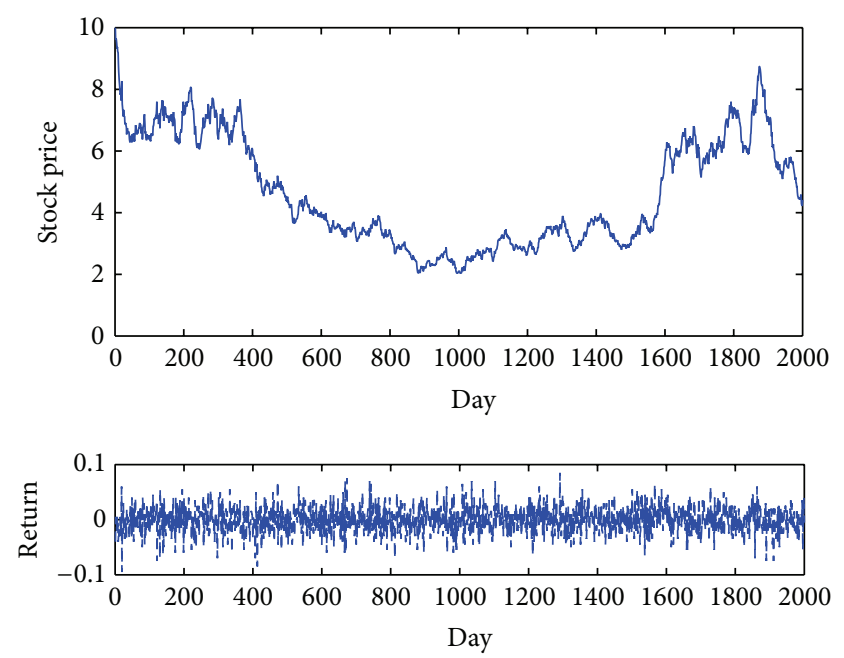

FIGURE 1: Fluctuations of prices and returns, respectively, for the financial price model.

The research results show that any $\mathrm{N}$-dimensional joint distribution function may be decomposed into $N$ marginal distributions, and the copula completely describes the dependence between the $N$ variables. Thus, the copula is a special multivariate distribution function which fully captures the dependence of the data used in many studies. The research of the present work is mainly concerned about the relationship between two variables. For any two-dimensional copula function $C(\cdot, \cdot)$, it should be satisfied with the following properties:

(1) $C(\cdot, \cdot):[0,1] \times[0,1] \rightarrow[0,1]$;

(2) $C(\cdot, \cdot)$ is grounded and two dimensional increasing, especially for all $u_{1}, u_{2}, v_{1}, v_{2} \in[0,1]$, when $u_{1}<$ $u_{2}, v_{1}<v_{2}$, then $C\left(u_{2}, v_{2}\right)-C\left(u_{2}, v_{1}\right)-C\left(u_{1}, v_{2}\right)+$ $C\left(u_{1}, v_{1}\right) \geq 0$

(3) for all variables $u, v \in[0,1], C(u, 1)=u, C(1, v)=v$, and $C(0, v)=C(u, 0)=0$.

In order to explore the dependence between two financial series, we introduce bivariate $\mathrm{t}$-copula in the following. The distribution function and density function of $\mathrm{t}$-copula can be written as follows [36, 37]:

$$
\begin{aligned}
C(u, v ; \rho, v)= & \int_{-\infty}^{T_{\nu}^{-1}(u)} \int_{-\infty}^{T_{\mu}^{-1}(v)} \frac{1}{2 \pi \sqrt{1-\rho^{2}}} \\
& \times\left[1+\frac{s^{2}+t^{2}-2 \rho s t}{\nu(1-\rho)^{2}}\right]^{-(\nu+2) / 2} d s d t, \\
c(u, v ; \rho, v)= & \rho^{-1 / 2} \frac{\Gamma((\nu+2) / 2) \Gamma(\nu / 2)}{[\Gamma((\nu+1) / 2)]^{2}} \\
& \times \frac{\left[1+\left(\varsigma_{1}^{2}+\varsigma_{2}^{2}-2 \rho \varsigma_{1} \varsigma_{2}\right) / \nu\left(1-\rho^{2}\right)\right]^{-(v+2) / 2}}{\Pi_{i=1}^{2}\left(1+\varsigma_{i}^{2} / \nu\right)^{-(v+2) / 2}},
\end{aligned}
$$

where $\rho \in(-1,1)$ denotes the linear correlation coefficient and $T_{\nu}^{-1}(\cdot)$ is the inverse function of one-dimensional 
t-distribution function with $\nu$ degrees, $\varsigma_{1}=T_{\mu}^{-1}(u), \varsigma_{2}=$ $T_{\mu}^{-1}(v)$.

\subsection{Tail Dependence and Concordance of Returns by t-Copula.} Tail dependence coefficient was introduced to reveal the effect on the other variable when one variable was given an extremely (comparatively) large or small value [38]. The survival function of copula $C(u, v)$ can be written as $\widehat{C}(u, v)$, whose definition is $\widehat{C}(u, v)=u+v-1+C(1-u, 1-v)$. By variable substitution, we can easily obtain $\widehat{C}(1-u, 1-v)=$ $1-u-v+C(u, v)$. For $u^{*} \in[0,1]$, we define [39]

$$
\psi\left(u^{*}\right)=P\left[U>u^{*} \mid V>u^{*}\right]=\frac{\widehat{C}\left(1-u^{*}, 1-u^{*}\right)}{1-u^{*}} .
$$

Here, $\psi\left(u^{*}\right)$ is called "quantile-dependent measure of dependence." We mainly consider the limit value of $\psi\left(u^{*}\right)$ when $u^{*} \rightarrow 1$. Provided $\lim _{u^{*} \rightarrow 1} \psi\left(u^{*}\right)$ exists, it can describe the properties of tail dependence. Further, for continuous random vector $(X, Y)$, let $F(x)$ and $G(y)$ represent its marginal distributions, respectively; we define

$$
\begin{aligned}
& \psi_{u}=\lim _{u \rightarrow 1} P\left[Y>G^{-1}(u) \mid X>F^{-1}(u)\right] \\
& =\lim _{u \rightarrow 1} \frac{\widehat{C}(1-u, 1-u)}{1-u}, \\
& \psi_{l}=\lim _{u \rightarrow 0} P\left[Y<G^{-1}(u) \mid X<F^{-1}(u)\right]=\lim _{u \rightarrow 0} \frac{C(u, u)}{u} \text {, }
\end{aligned}
$$

where $\psi_{u}$ is the upper dependence coefficient between $X$ and $Y$ and $\psi_{l}$ is the lower dependence coefficient between $X$ and $Y$. If $\psi_{u}$ (or $\left.\psi_{l}\right)$ exists and has positive value $(0<$ $\psi_{u}, \psi_{l} \leq 1$ ), we say random variables $X, Y$ are upper (or lower) asymptotically dependent or correlated. When $\psi_{u}$ (or $\psi_{l}$ ) is equal to zero, we say $X, Y$ are upper (or lower) asymptotically independent. For an intuitive aspect, the upper tail dependence coefficient $\psi_{u}$ stands for the probability of two financial markets' turning up an extremely large price simultaneously, and the lower tail dependence coefficient $\psi_{l}$ represents the probability of two financial markets' turning up an extremely small price simultaneously. It is clear that, for analysis in financial markets and financial assets, tail dependence coefficient of copula will be quite convenient and effective. For example, we can understand whether a slump in one stock price results in a slump in another stock price by studying the behavior of the tail dependence coefficient which is greatly helpful for the explanation on volatility spillover in financial markets.

In addition to tail dependence coefficient, there are several kinds of measures of correlations with respect to t-copula function, for example, Kendall order correlation coefficient [40] and Spearman order correlation coefficient [41]. Assume $\left(x_{1}, y_{1}\right),\left(x_{2}, y_{2}\right)$ are the observed values of random vector $(X, Y)$, and we say $\left(x_{1}, y_{1}\right)$ and $\left(x_{2}, y_{2}\right)$ are concordant if $\left(x_{1}-x_{2}\right)\left(y_{1}-y_{2}\right)>0$ and otherwise discordant for $\left(x_{1}-\right.$ $\left.x_{2}\right)\left(y_{1}-y_{2}\right)<0$. Two mutual independent two-dimensional random vectors $\left(X_{1}, Y_{1}\right),\left(X_{2}, Y_{2}\right)$ have the same distribution as $(X, Y)$; let $P\left[\left(X_{1}-X_{2}\right)\left(Y_{1}-Y_{2}\right)>0\right]$ denote the probability of their concord and $P\left[\left(X_{1}-X_{2}\right)\left(Y_{1}-Y_{2}\right)<0\right]$ denote the probability of their discord. The difference between concordant and discordant probability is the Kendall order correlation coefficient $\kappa$. Suppose $\left(X_{i}, Y_{i}\right)(i=1,2, \ldots, n)$ are the samples from $(X, Y) ; c$ and $d$ denote the observed numbers of concord and discord. Hence, we get the samples' Kendall order correlation coefficient

$$
\kappa=\frac{c-d}{c+d}=\frac{c-d}{\left(\begin{array}{l}
n \\
2
\end{array}\right)} .
$$

Generally, $\left(x_{1}, y_{1}\right),\left(x_{2}, y_{2}\right)$ are independent and identically distributed vectors; $\kappa$ is defined as follows:

$$
\begin{aligned}
\kappa \equiv & P\left[\left(x_{1}-x_{2}\right)\left(y_{1}-y_{2}\right)>0\right] \\
& -P\left[\left(x_{1}-x_{2}\right)\left(y_{1}-y_{2}\right)<0\right] .
\end{aligned}
$$

We can judge the degree of correlation between random variable $X$ and $Y$ from the definition of $\kappa$. When $\kappa=1$, $X$ varies in full accord with $Y$ and they are positive crosscorrelated; when $\kappa=-1, X$ varies in full opposite accord with $Y$ and they are negative cross-correlated; when $\kappa=0$, the variation between $X$ and $Y$ is half in accord and half in opposite accord, indicating an unclear correlation between $X$ and $Y$.

Suppose $\left(X_{1}, Y_{1}\right),\left(X_{2}, Y_{2}\right),\left(X_{3}, Y_{3}\right)$ are two-dimensional random vectors with mutual independence and have the same distribution as $(X, Y)$. We define Spearman order correlation coefficient as follows:

$$
\begin{aligned}
\rho_{s}=3\{ & P\left[\left(X_{1}-X_{2}\right)\left(Y_{1}-Y_{3}\right)>0\right] \\
- & \left.P\left[\left(X_{1}-X_{2}\right)\left(Y_{1}-Y_{3}\right)<0\right]\right\} ;
\end{aligned}
$$

$\left(X_{i}, Y_{i}\right)(i=1,2, \ldots, n)$ are samples from $(X, Y)$, let $R_{i}$ represent the order of $X_{i}$ in $\left(X_{1}, X_{2}, \ldots, X_{n}\right)$, and let $Q_{i}$ represent the order of $Y_{i}$ in $\left(Y_{1}, Y_{2}, \ldots, Y_{n}\right)$. Then the samples' Spearman order correlation coefficient is

$$
\rho_{s}=\frac{\sum_{i=1}^{n}\left(R_{i}-\bar{R}\right)\left(Q_{i}-\bar{Q}\right)}{\sqrt{\sum_{i=1}^{n}\left(R_{i}-\bar{R}\right)^{2}} \sqrt{\sum_{i=1}^{n}\left(Q_{i}-\bar{Q}\right)^{2}}},
$$

where $\bar{R}=(1 / n) \sum_{i=1}^{n} R_{i}, \bar{Q}=(1 / n) \sum_{i=1}^{n} Q_{i}$. By simplification, we can obtain

$$
\rho_{s}=1-\frac{6}{n\left(n^{2}-1\right)} \sum_{i=1}^{n}\left(R_{i}-Q_{i}\right)^{2}
$$

In the introductions of tail dependence coefficient $\left(\psi_{u}\right.$ and $\psi_{l}$ ), Kendall order correlation coefficient $\kappa$ and Spearman order coefficient $\rho_{s}$ in $\mathrm{t}$-copula method interpret the strength of tail dependence and concordance among the real markets and the financial model. It is possible to evaluate the possibility for two financial markets' turning up an extremely large (small) value simultaneously, which is of great help in fields of asset pricing and financial risk management. 


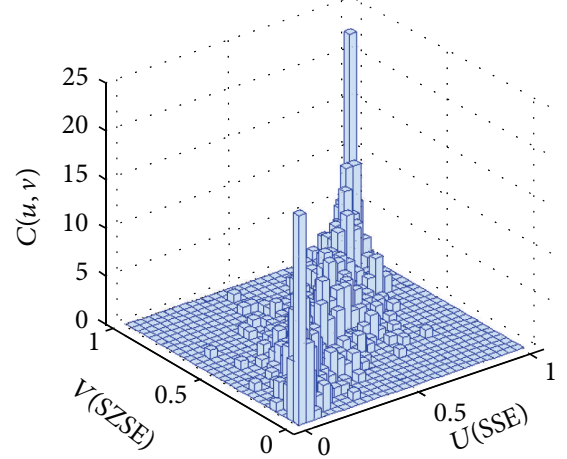

(a)

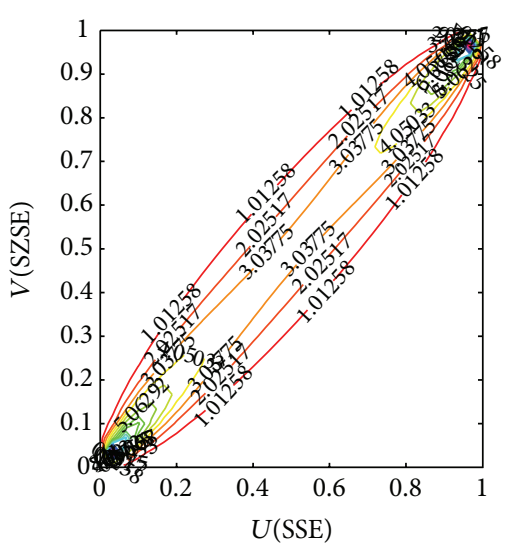

(c)

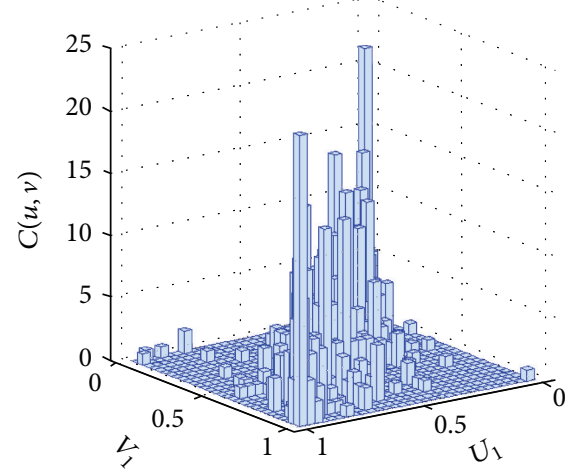

(e)

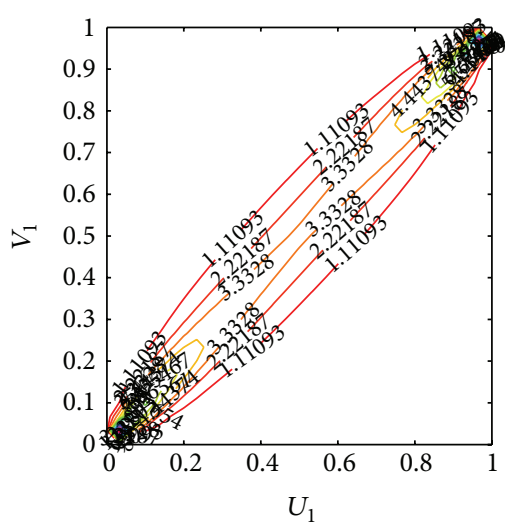

(g)

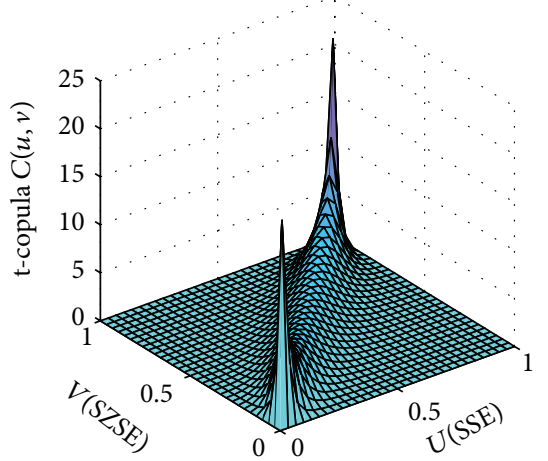

(b)

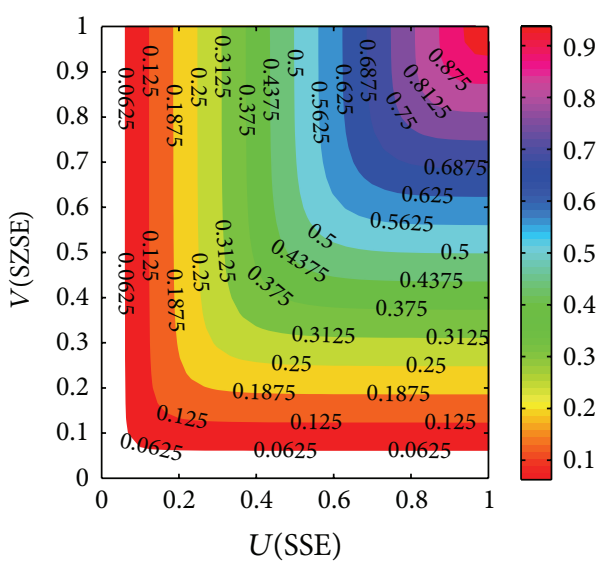

(d)

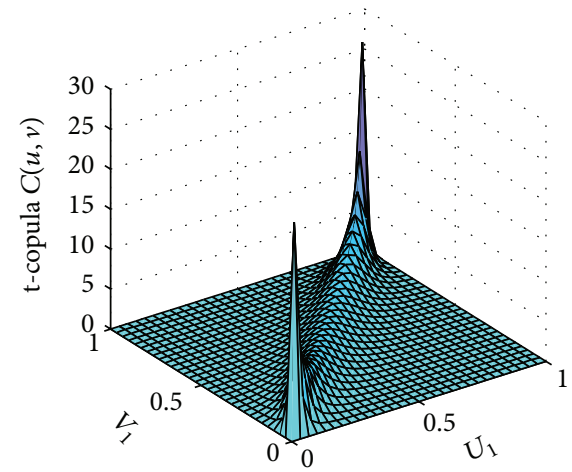

(f)

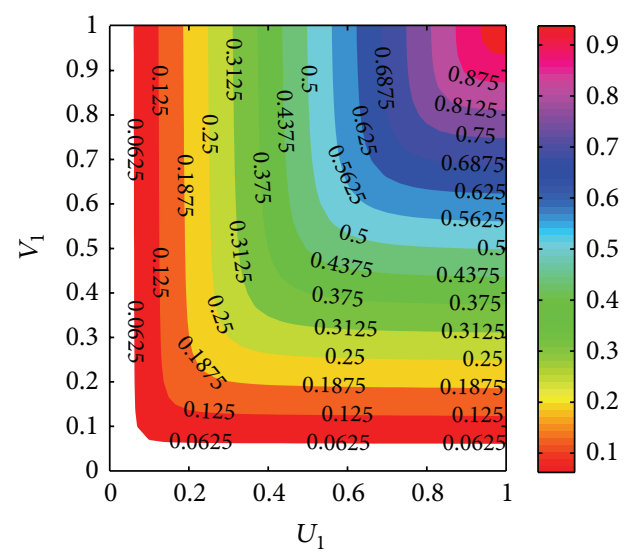

(h)

FIGURE 2: Continued. 


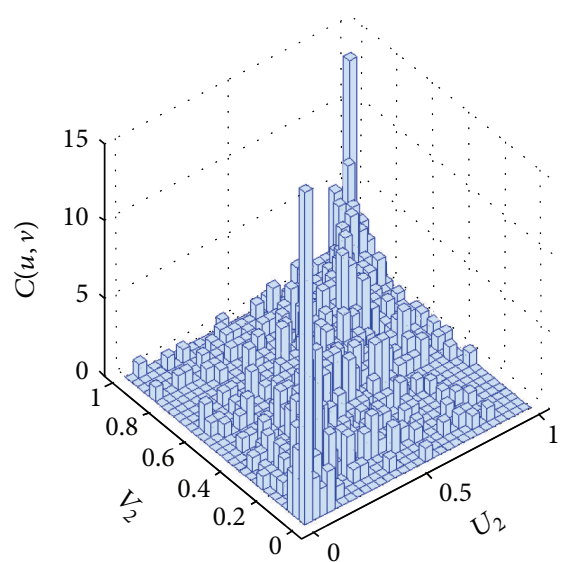

(i)

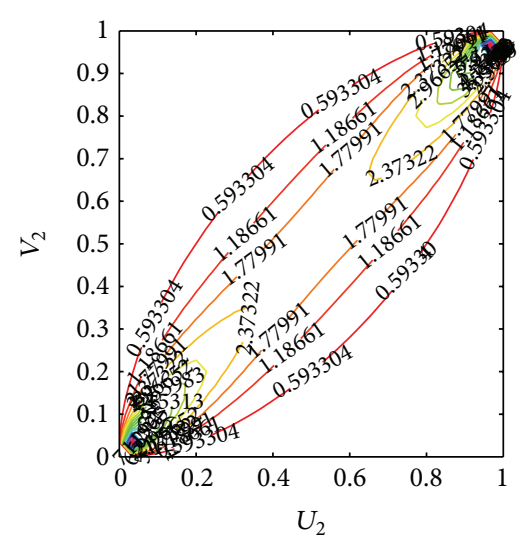

$(\mathrm{k})$

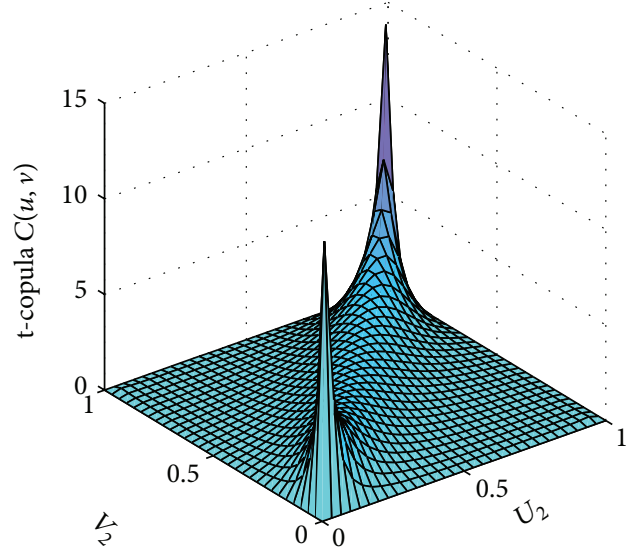

$(\mathrm{j})$
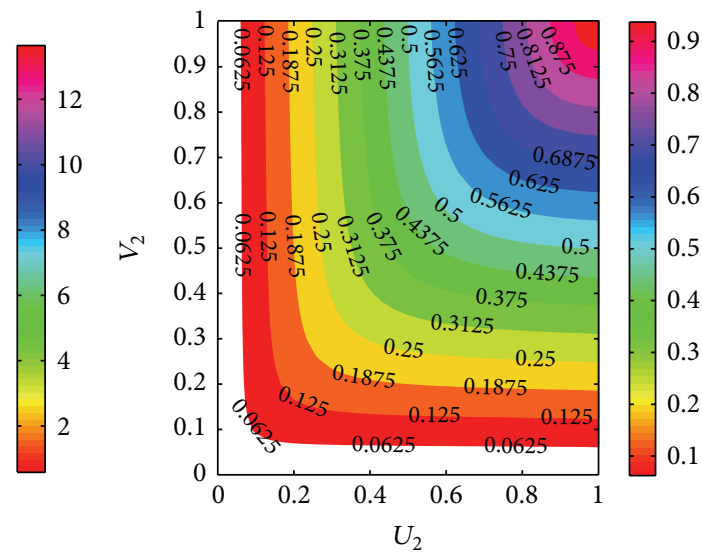

(1)

FIGURE 2: Plots of SSE and SZSE indexes by t-copula. (a) Frequency histogram; (b) density function diagram; (c) contour of joint density functions; (d) contour of distribution functions. Plots of the model with S.datal $(\lambda=2$ and $\lambda=2.5)$ by t-copula. (e) Frequency histogram; (f) density function diagram; (g) contour of joint density functions; (h) contour of distribution functions. Plots of the model with S.datal $(\lambda=3$ and $\lambda=2.5$ ) by t-copula. (i) Frequency histogram; (j) density function diagram; (k) contour of joint density functions; (l) contour of distribution functions.

3.3. Empirical Results of t-Copula Analysis. In this part, we make an empirical research on the tail dependence of real data and simulation data by t-copula function. The empirical data comes from intraday data of SSE and SZSE from April 2, 2009, to May 9, 2013, and two pairs of simulated data are generated from the proposed model with different parameter values (S.datal: $\lambda=2$ and $\lambda=2.5$ and S.data2: $\lambda=3$ and $\lambda=2.5)$. We select the proper parameter values by Monte Carlo method above the threshold of financial model and ensure these two pairs of simulative financial time series close to the real markets data in some degree. Figure 2 shows the plots of frequency histogram, density function diagram, contour of joint density functions, and contour of distribution functions for both actual data (SSE and SZSE) and two pairs of simulated data $(\lambda=2$ and $\lambda=2.5$ and $\lambda=3$ and $\lambda=2.5$ ). From Figures 2(a), 2(e), 2(i), 2(b), $2(\mathrm{f})$, and $2(\mathrm{j})$, the plots exhibit that there exists relatively strong tail dependence by $\mathrm{t}$-copula in three groups of data, which demonstrate the dependence in the actual data and the proposed model. By comparing the contours of Figures 2(c), $2(\mathrm{~g})$, and $2(\mathrm{k})$ of joint density functions, we find that S.datal $(\lambda=2$ and $\lambda=2.5)$ is closer to the real market and has a larger peak height and faster converge than S.data2 $(\lambda=3$ and $\lambda=2.5$ ). From Figures $2(\mathrm{~d}), 2(\mathrm{~h})$, and 2(l), we have analogical features about the contours of distribution functions for three groups of data and a better understanding of the t-copula distribution functions from another aspect.

For further evidence and explanation about the tail dependence, we give the corresponding measures by $\mathrm{t}$-copula in Table 1. From Table 1, the values of Kendall and Spearman coefficients for SSE and SZSE are larger than those of S.datal and S.data2, stating a stronger property of concordance in the real market data itself and better correlations between two Chinese stock markets. It also indicates that SSE and SZSE simultaneously go up and fall down for most of time. According to the symmetrical character of t-copula, the upper tail dependence coefficient $\psi_{u}$ is equal to the lower tail dependence coefficient $\psi_{l}$. For the tail dependence coefficients of three pairs of data, all of them are positive values, showing a positive upper and lower tail correlated 
TABLE 1: Measures for concordance and tail dependence of returns.

\begin{tabular}{lcccc}
\hline Data & $\kappa$ & $\rho_{s}$ & $\psi_{l}$ & $\psi_{u}$ \\
\hline SSE and SZSE & 0.7871 & 0.9395 & 0.7384 & 0.7384 \\
Pair of S.data1 & 0.7546 & 0.9200 & 0.6937 & 0.6937 \\
Pair of S.data2 & 0.6153 & 0.8099 & 0.5941 & 0.5941 \\
\hline
\end{tabular}

phenomenon. The actual one (SSE and SZSE) with the biggest upper and lower tail dependence coefficient means the closely positive correlation between two stock markets in China. In an intuitive way, we can say that two real Chinese stock markets' (SSE and SZSE) turning up an extremely large or small value simultaneously has a possibility of 0.7384 and is larger than the possibilities of two pairs of simulative markets (0.6937 and 0.5941), clearly showing the strong tail dependence and concordance among them and providing a new idea and decision making in the fields of asset pricing and financial risk management. In the meantime, the simulation data (S.datal and S.data2) of the proposed model present the similar features of concordance and tail dependence as well, demonstrating the rationality and feasibility of the proposed model.

\section{Methodology for Statistical Analysis}

4.1. Test for Cross-Correlation. There have developed several statistical methods aiming at demonstrating and quantifying the cross-correlation from the aspects of detrended crosscorrelation and power-law cross-correlation [42]. Here, we use a cross-correlation statistic put forward by Podobnik et al. [43] to testify and quantify the cross-correlation in Chinese markets (SSE and SZSE) and financial model data. Below is the test for the existence of cross-correlation between two time series. Suppose $\{x(i)\}$ and $\{y(i)\}$ are two financial time series with the same length of $N$. We have the relationship as follows:

$$
C_{i}=\frac{\sum_{k=i+1}^{N} x_{k} y_{k-i}}{\sqrt{\sum_{k=1}^{N} x_{k}^{2} \sum_{k=1}^{N} y_{k}^{2}}} .
$$

Thus, we can obtain the cross-correlation statistic

$$
Q_{c c}(m)=N^{2} \sum_{i=1}^{m} \frac{C_{i}^{2}}{N-i} .
$$

The cross-correlation statistic $Q_{c c}(m)$ complies well with the $\chi_{(m)}^{2}$ distribution if there exists no cross-correlation between two time series. If the value of cross-correlations statistic $Q_{c c}(m)$ exceeds the critical value of the $\chi_{(m)}^{2}$ distribution, indicating significant cross-correlations at a significance level, we present the critical value of the $\chi_{(m)}^{2}$ distribution and relevant cross-correlation statistic $Q_{c c}(m)$ for the three pairs of time series at the $5 \%$ level of significance with the degrees of freedom ranging from 1 to 600 in Figure 3.

From Figure 3, we can clearly see these three pairs of data all exceeding the critical value of the $\chi_{(m)}^{2}$ distribution at the $5 \%$ level of significance, suggesting the existence of

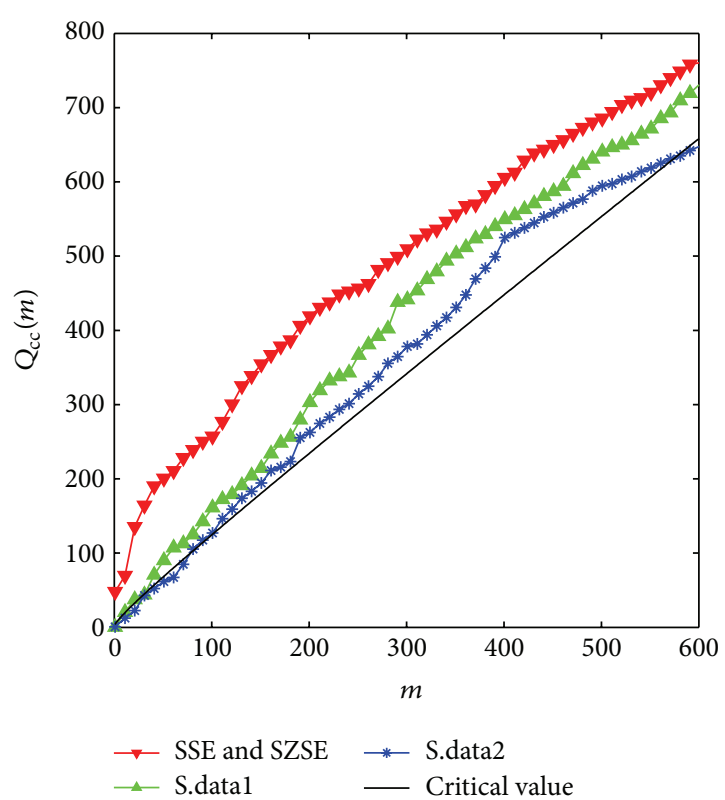

FIgURE 3: The cross-correlation statistic $Q_{c c}(m)$ of return series for the real markets and the financial model.

cross-correlation in both real stock markets and proposed financial model. In some aspect, it also reflects the rationality and feasibility in proposed financial model. Further, we also can figure out the strength of cross-correlation by the distance between the critical value line and $Q_{c c}(m)$. The strength of cross-correlation between SSE and SZSE is obviously stronger than another two pairs of data in proposed model, and the first simulative pair's (S.datal) cross-correlation is stronger than the second pair's (S.data2).

4.2. MF-DCCA for Financial Time Series. In this section, we apply the multifractal detrended cross-correlation analysis (MF-DCCA) to investigating the cross-correlation of returns with multifractality $[10,11,13]$. Suppose two time series $\{x(t)\}$ and $\{y(t)\}(t=1, \ldots, N)$ with the same length of $N$. Firstly, we calculate the "profiles" of $\{x(t)\}$ and $\{y(t)\}$

$$
X(i)=\sum_{k=1}^{i}\left[x_{k}-\bar{x}\right], \quad Y(i)=\sum_{k=1}^{i}\left[y_{k}-\bar{y}\right],
$$

where $\bar{x}$ and $\bar{y}$ denote the average of two whole time series $\{x(t)\}$ and $\{y(t)\}$, respectively. Then, we separate $X(i)$ and $Y(i)$ into $N_{s}=[N / s]$ nonoverlapping segments with an equal length $s$ ( $s$ is often called time window). We perform the same process as well from the other end to make sure that the whole series could be considered as far as possible. Thus, we can get $2 N_{s}$ segments with the equal length $s$. We estimate the local trends for each of the $2 N_{s}$ segments by an $m$ th-order polynomial fit. The corresponding detrended covariance is provided as follows, for $v=1,2, \ldots, N_{s}$,

$$
\begin{aligned}
F_{\mathrm{DCCA}}^{2}(s, v)=\frac{1}{s} \sum_{j=1}^{s} & \left|X((v-1) s+j)-X^{v}(j)\right| \\
\times & \left|Y((v-1) s+j)-Y^{v}(j)\right|
\end{aligned}
$$


and for $v=N_{s}+1, N_{s}+2, \ldots, 2 N_{s}$

$$
\begin{aligned}
F_{\mathrm{DCCA}}^{2}(s, v)=\frac{1}{s} \sum_{j=1}^{s} & \left|X\left(N-\left(v-N_{s}\right) s+j\right)-X^{v}(j)\right| \\
\times & \left|Y\left(N-\left(v-N_{s}\right) s+j\right)-Y^{v}(j)\right| .
\end{aligned}
$$

The trends $X^{v}(j), Y^{v}(j)$ are the fitting polynomials with order $m$ in segment $v$. Then average overall segments to obtain the $q$ th-order fluctuation function

$$
\begin{aligned}
& F_{q}(s)=\left\{\frac{1}{2 N_{s}} \sum_{v=1}^{2 N_{s}}\left[F_{\mathrm{DCCA}}^{2}(s, v)\right]^{q / 2}\right\}^{1 / q}, \\
& F_{0}(s)=\exp \left\{\frac{1}{4 N_{s}} \ln \left[F_{\mathrm{DCCA}}^{2}(s, v)\right]\right\} .
\end{aligned}
$$

We analyze the scaling behavior by studying log-log plots of $F_{q}(s)$ versus $s$ for each value of $q$. Two long-range crosscorrelated series exhibit a power-law expression: $F_{q}(s) \propto$ $s^{h_{x y}(q)}$. The power-law relationship between the two correlated series can be described by $h_{x y}(q)$. If $h_{x y}(q)$ depends on $q$, we say that there exists the phenomenon of multifractality. If the cross-correlation exponent $h_{x y}(q)>0.5$, the crosscorrelations between the return fluctuations of two series related $q$ are persistent (positive), which implies that one market is likely to increase (decrease) following an increase (decrease) of the other market. If $h_{x y}(q)<0.5$, the crosscorrelations between the return fluctuations of two series related $q$ are antipersistent (negative), which implies that one market is likely to increase (decrease) following a decrease (increase) of the other market. These two situations above illustrate the persistent (positive) or antipersistent (negative) cross-correlation between two financial markets, which do not conform to the concept and definition of Brownian fluctuation, leading to the violation of the well-known market efficiency hypothesis put forward by Fama [44]. If $h_{x y}(q)=$ 0.5 , there are no cross-correlations between two series and the volatility of one market may not influence the other market evidently. At the same time, the price changes cannot be predicted and comply with the Brownian motion (or random walk), which is consistent with the market efficiency hypothesis. The Hurst exponent also provides a measure to determine the speed of prices' adjustment to a random walk, namely, recovering to an efficient market $[45,46]$.

In particular, when the time series $\{x(t)\}$ is identical to $\{y(t)\}$, it is clear that

$$
\begin{aligned}
F_{\mathrm{DCCA}}^{2}(s, v) & =F_{\mathrm{DFA}}^{2}(s, v), \\
F_{q}(s) & =\left\{\frac{1}{2 N_{s}} \sum_{v=1}^{2 N_{s}}\left[F_{\mathrm{DFA}}^{2}(s, v)\right]^{q / 2}\right\}^{1 / q} ;
\end{aligned}
$$

MF-DCCA is retrieved to MF-DFA. When $q=2$,

$$
F_{q}(s)=\left\{\frac{1}{2 N_{s}} \sum_{v=1}^{2 N_{s}}\left[F_{\text {DCCA }}^{2}(s, v)\right]\right\}^{1 / 2} ;
$$

MF-DCCA is reduced to DCCA. The cross-correlation exponent $h_{x y}(q)$ is equivalent to the generalized Hurst exponent $h_{x y}(2)$. The similar relationships between classical multifractal scaling exponents can be shown as follows: $\omega_{x y}(q)=$ $q h_{x y}(q)-1$. If $\omega_{x y}(q)$ is linear with $q$, the cross-correlation of correlated series is monofractal; otherwise it is multifractal. By the Legendre transformation [11], we can obtain the following relationships: $\alpha=h_{x y}(q)+q h_{x y}^{\prime}(q), f_{x y}(\alpha)=$ $q\left[\alpha-h_{x y}(q)\right]+1$, where $h_{x y}^{\prime}(q)$ denotes the derivative of $h_{x y}(q)$ with respect to $q$ and $\alpha$ is the Hölder exponent or singularity strength, which characterizes the singularities in the time series. For the situation of multifractality, different portions are characterized by different $\alpha$, causing the existence of spectrum $f(\alpha)$. The strength of multifractality can be estimated by the width of multifractal spectrum, which is given by $\Delta \alpha=\alpha_{\max }-\alpha_{\min }$. The difference of the fractal dimensions of the maximum probability subset $\left(\alpha=\alpha_{\text {min }}\right)$ and the minimum one $\left(\alpha=\alpha_{\max }\right)$ is as follows [47]:

$$
\Delta f=f\left(\alpha_{\min }\right)-f\left(\alpha_{\max }\right) .
$$

Further, we give the concept of fractal asymmetric exponent FAE $=\left(\Delta \alpha_{l}-\Delta \alpha_{r}\right) /\left(\Delta \alpha_{l}+\Delta \alpha_{r}\right)$, in which $\Delta \alpha_{l}$ and $\Delta \alpha_{r}$ are defined as $\Delta \alpha_{l}=\widehat{\alpha}-\alpha_{\min }$ and $\Delta \alpha_{r}=\alpha_{\max }-\widehat{\alpha}$. Here, $\widehat{\alpha}$ is the value on condition that $f(\widehat{\alpha})=\max f(\alpha)$. By this formulation, we can understand that the larger value of $\Delta \alpha$ will lead to the wider probability distribution and larger difference between the highest stock price and lowest stock price.

The application of MF-DCCA method aims at considering the cross-correlation for both real data and simulated data in an angle of multifractality different from copula theory mentioned in Section 3. The generalized Hurst exponent illustrates the multifractal positive (persistent) crosscorrelation in Chinese stock markets along with the financial model and provides an evidence of the market inefficiency. Also, the strength of multifractal spectra $f(\alpha)$ implies the proof for the inefficiency of financial market which violates the well-known market efficiency hypothesis [44].

4.3. Empirical Research by MF-DCCA Analysis. The multifractal detrended cross-correlations between two return series by MF-DCCA are displayed in Figure 4 . There are 21 empirical curves in each plot and they emerge in a descending order of $q(q=-10,-9, \ldots, 9,10)$ from bottom to top, which suggests that return series for both the real markets and the simulation data display the similar character of power-law scaling, sharing the power-law scaling property of fluctuation function with each other. It may provide a sign of multifractal detrended cross-correlations that a large increment of price changes in one market may be more likely to be followed by a large increment of price changes in another geographically or temporally correlated market.

Figure 5 shows the tendency of cross-correlation exponents $h_{x y}(q)$ of return series for SSE and SZSE as well as the proposed model. It also exhibits that the value of crosscorrelation exponent $h_{x y}(q)$ decreases when $q$ increases. If the cross-correlation exponent $h_{x y}(q)$ is a constant, the market is monofractal; otherwise it is multifractal. These plots 

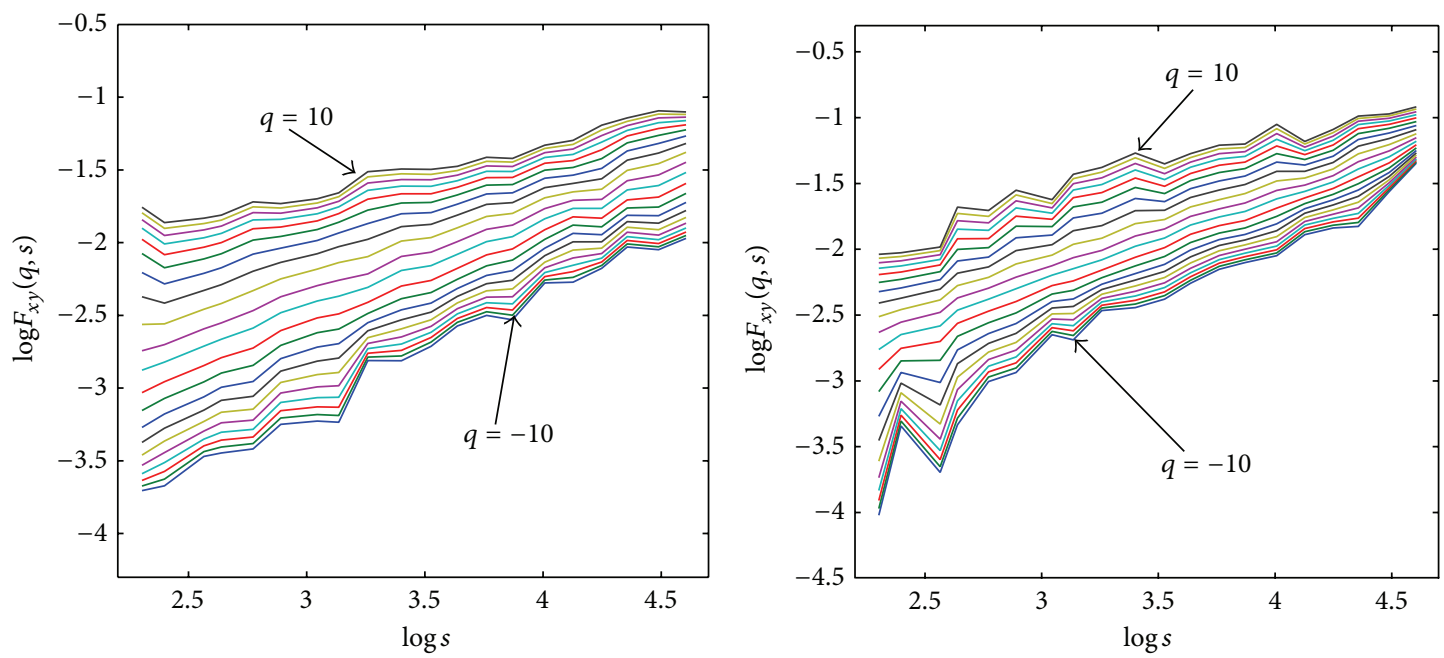

(a)

(b)

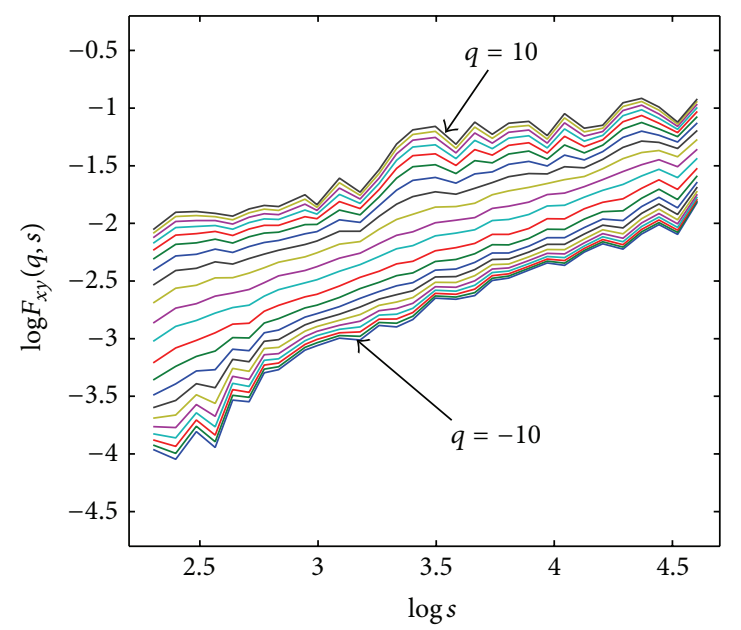

(c)

Figure 4: Log-log plots of $F_{x y}(q, s)$ versus $s$ for (a) the real markets and (b), (c) the proposed model with S.datal and S.data2, respectively.

show that the cross-correlation relationships are nonlinear and multifractal, since there are dissimilar exponents $h$ for different values of $q$. Figure 6 exhibits the plots of multifractal scaling exponents $\omega_{x y}(q)$ of return series for the real markets and the proposed model. The multifractal exponents $\omega$ is nonlinearly dependent on $q$, which provides the empirical evidence that the multifractality exists in three pairs of time series.

The corresponding detailed values of cross-correlation exponent $h_{x y}(q)$ and multifractal scaling exponent $\omega_{x y}(q)$ for the real markets and the proposed model are displayed in Table 2. Table 2 shows the monotone diminution of $h_{x y}(q)$ and the monotone nonlinear increase of $\omega_{x y}(q)$ with the increasing of $q$, reflecting the apparent multifractal property of cross-correlation for actual and simulated data. When $q<4$, the cross-correlation exponent $h_{x y}(q)>0.5$ for the real data, there has a persistent (positive) cross-correlation between SSE and SZSE, stating that the distinct rise and fall for SSE may be followed as the same type in SZSE later. When $q=2$, the generalized Hurst exponent $h_{x y}(q)$ of real markets is also greater than 0.5 (0.5766), showing a weak persistent (positive) cross-correlation between two stock markets. When $q>4, h_{x y}(q)<0.5$ displays an antipersistent (negative) cross-correlation, signifying that the distinct rise and fall for SSE may be followed as the opposite type in SZSE later. The similar explanations above can go right with regard to the model data (S.datal and S.data2). From the viewpoint of market efficiency hypothesis, we can judge that the cross-correlation does not comply with the random walk [46] at the situation of $q<4$ and $q>4$, illustrating a fact of market inefficiency. Only when $q$ is around $4\left(h_{x y}\right.$ is about 0.5$)$, the cross-correlation is 
TABLE 2: Cross-correlation exponent $h_{x y}(q)$ and multifractal scaling exponent $\omega_{x y}(q)$.

\begin{tabular}{|c|c|c|c|c|c|c|}
\hline \multirow{2}{*}{$\begin{array}{l}\text { Data } \\
q\end{array}$} & \multicolumn{3}{|c|}{ Cross-correlation exponent $h_{x y}(q)$} & \multicolumn{3}{|c|}{ Multifractal scaling exponent $\omega_{x y}(q)$} \\
\hline & R.data & S.datal & S.data2 & R.data & S.datal & S.data2 \\
\hline-10 & 0.8664 & 0.8425 & 0.8076 & -8.8641 & -8.8248 & -8.5758 \\
\hline-9 & 0.8569 & 0.8321 & 0.8014 & -7.9922 & -7.9489 & -7.7622 \\
\hline-8 & 0.8456 & 0.8196 & 0.7942 & -7.1247 & -7.0772 & -6.9537 \\
\hline-7 & 0.8320 & 0.8045 & 0.7820 & -6.2643 & -6.2117 & -6.1520 \\
\hline-6 & 0.8158 & 0.7860 & 0.7665 & -5.4148 & -5.3562 & -5.3592 \\
\hline-5 & 0.7965 & 0.7634 & 0.7479 & -4.5824 & -4.5171 & -4.5782 \\
\hline-4 & 0.7739 & 0.7364 & 0.7281 & -3.7756 & -3.7055 & -3.8123 \\
\hline-3 & 0.7483 & 0.7060 & 0.7035 & -3.0049 & -2.9379 & -3.0654 \\
\hline-2 & 0.7200 & 0.6748 & 0.6800 & -2.2800 & -2.2296 & -2.3420 \\
\hline-1 & 0.6891 & 0.6447 & 0.6551 & -1.6091 & -1.5847 & -1.6490 \\
\hline 0 & 0.6507 & 0.6143 & 0.6252 & -1 & -1 & -1 \\
\hline 1 & 0.6172 & 0.5870 & 0.5972 & -0.4628 & -0.4730 & -0.4128 \\
\hline 2 & 0.5766 & 0.5590 & 0.5602 & -0.0067 & -0.0020 & 0.1004 \\
\hline 3 & 0.5370 & 0.5321 & 0.5241 & 0.3711 & 0.4163 & 0.5423 \\
\hline 4 & 0.5024 & 0.5072 & 0.4917 & 0.6898 & 0.7887 & 0.9269 \\
\hline 5 & 0.4744 & 0.4848 & 0.4642 & 0.9722 & 1.1242 & 1.2710 \\
\hline 6 & 0.4524 & 0.4653 & 0.4414 & 1.2346 & 1.4321 & 1.5886 \\
\hline 7 & 0.4351 & 0.5486 & 0.4386 & 1.4858 & 1.7204 & 1.8893 \\
\hline 8 & 0.4213 & 0.5344 & 0.4074 & 1.7306 & 1.9953 & 2.1793 \\
\hline 9 & 0.4102 & 0.4224 & 0.3947 & 1.9715 & 2.2614 & 2.4622 \\
\hline 10 & 0.4010 & 0.4122 & 0.3841 & 2.2097 & 2.5216 & 2.7405 \\
\hline
\end{tabular}

Note: R.data means the real data; S.datal and S.data2 denote two pairs of simulated data.

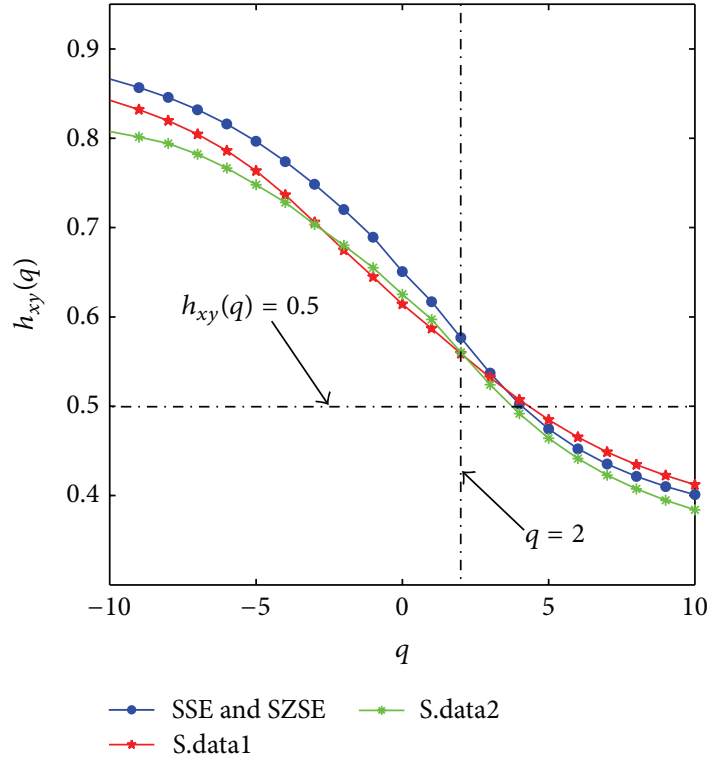

Figure 5: Cross-correlation exponent $h(q)$ of return series for the real markets and the financial model.

consistent with Brownian fluctuation, supporting the market efficiency hypothesis [44]. In order to realize the multifractal character of two financial time series, we study multifractal scaling exponent $\Phi_{x y}(q)$ and observe its specific numerical values. From Table 2 , both the actual and simulated data

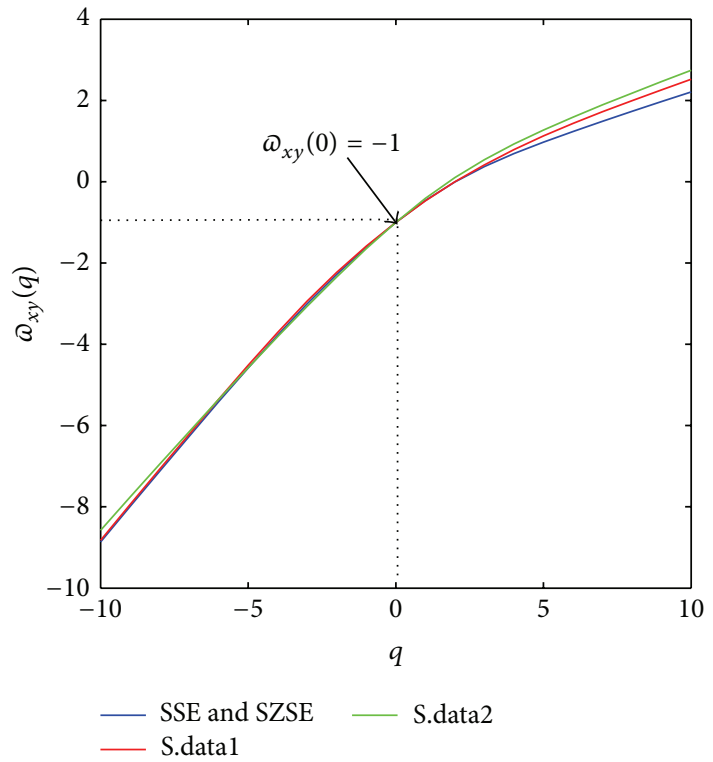

FIGURE 6: Multifractal scaling exponent $\omega(q)$ of return series for the real markets and the financial model.

present the trend of increase with respect to $q$ in a nonlinear way, uncovering the multifractality of cross-correlation. In addition, the three pairs of data share the same value $\omega_{x y}(q)=$ -1 at the point $q=0$, which agrees with the relevant theory and the coincident point is also labeled in Figure 6. 
TABLE 3: Multifractal spectra for real markets and simulated data.

\begin{tabular}{lcccc}
\hline Data & $\Delta \alpha_{l}$ & $\Delta \alpha_{r}$ & $\Delta f$ & FAE \\
\hline SSE and SZSE & 0.3708 & 0.2628 & 0.0278 & 0.1705 \\
Pair of S.datal & 0.3245 & 0.2912 & 0.0142 & 0.0541 \\
Pair of S.data2 & 0.3707 & 0.1673 & -0.3967 & 0.3780 \\
\hline
\end{tabular}

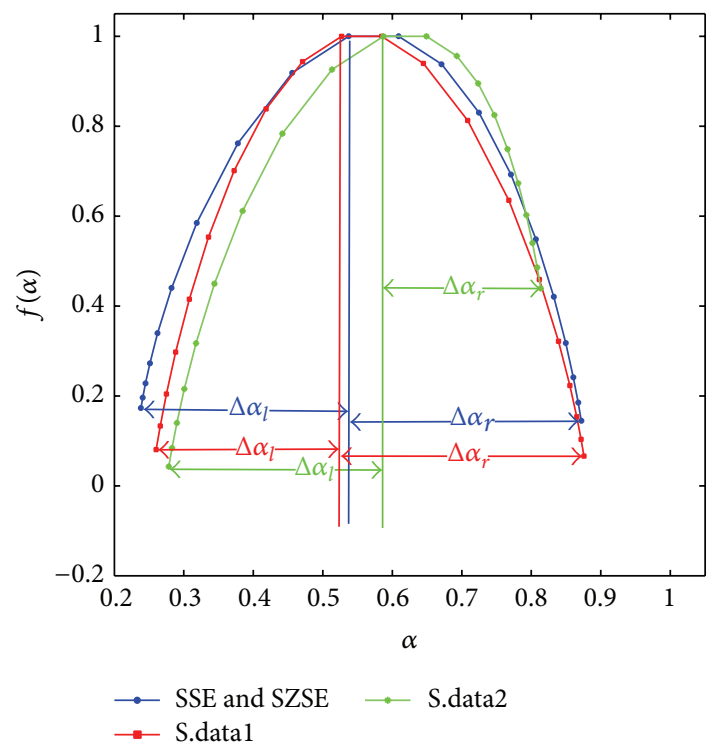

FIGURE 7: Multifractal spectra $f(\alpha)$ of returns for the real data and the simulation data.

Now we study the multifractal strength by the multifractal spectra method. A system is monofractal if the multifractal spectrum of monofractality is a point; that is, the width of multifractal spectrum is zero. In fact, the width of multifractal spectrum can be regarded as a measure of multifractal strength. In Figure 7, the widths of crosscorrelation multifractal spectral for the return series are significantly nonzero, which illustrate that there exist clearly departures from random walk process for cross-correlated return series. Table 3 gives the multifractal strength of the real data and the simulation data. We notice the three pairs of data are satisfied with $\Delta \alpha_{l}>\Delta \alpha_{r}$. In addition, R.data (SSE and SZSE) and S.datal have approximate values of $\Delta \alpha_{l}$ and $\Delta \alpha_{r}$, indicating the multifractal strength of cross-correlation in real markets is similar to that in the first simulation data (S.datal). However, S.data2 has a weaker multifractal strength with respect to R.data and S.datal for its smaller $\Delta \alpha$. As to the fractal asymmetric exponent FAE, not only the actual markets but also the model are all greater than zero, and the value of FAE of the R.data is between those of S.datal and S.data2.

By MF-DCCA method, we also detect the existence of multifractality of cross-correlation in both real and simulative markets. The measures of strength of multifractality $\Delta \alpha\left(\Delta \alpha_{l}+\Delta \alpha_{r}\right)$ of real and simulative markets are 0.6336, 0.6157 , and 0.5380 , respectively, implying the evidence that a stronger multifractality is accompanied with a more inefficient market [45]. From the results in Table 3, the real financial markets (SSE and SZSE) have the strongest multifractality of cross-correlation together with the most inefficiency (also the farthest from the random walk or Brownian motion) among all pairs of data.

\section{Conclusion}

In the present paper, we analyze tail dependence and multifractal detrended cross-correlations of return series between two real stock market indexes (SSE and SZSE) by copula and MF-DCCA method, respectively. And we also introduce a stock price process by applying the voter interacting dynamic system to perform empirical research for comparisons with the actual data. The empirical results show that the return series for both the actual data and the simulated data have the property of positive upper and lower tail dependence, multifractality, and cross-correlation. The results supply an intuitive possibility to display the tail dependence coefficient of two financial markets which is helpful in the fields of asset pricing and financial risk management and also obtain an intuitive evidence of market inefficiency from the view of multifractality. Through the comparisons between the real markets and the proposed model, we hope to show that the financial model of the present work is reasonable to some extent.

\section{Conflict of Interests}

The authors declare that there is no conflict of interests regarding the publication of this paper.

\section{Acknowledgment}

The authors were supported in part by National Natural Science Foundation of China Grant no. 71271026 and Grant no. 10971010.

\section{References}

[1] P. Gopikrishnan, V. Plerou, L. A. N. Amaral, M. Meyer, and H. E. Stanley, "Scaling of the distribution of fluctuations of financial market indices," Physical Review E, vol. 60, no. 5, pp. 5305-5316, 1999.

[2] A. Pagan, "The econometrics of financial markets," Journal of Empirical Finance, vol. 48, no. 1-3, pp. 15-102, 1996.

[3] R. N. Mantegna and H. E. Stanley, "Scaling behaviour in the dynamics of an economic index," Nature, vol. 376, no. 6535, pp. 46-49, 1995.

[4] M. Sklar, "Fonctions de répartition à $n$ dimensions et leurs marges," vol. 8, pp. 229-231, 1959.

[5] R. B. Nelsen, An Introduction to Copula, Elsevier Science, New York, NY, USA, 1983.

[6] H. E. Hurst, "Long term storage capacity of reservoirs," Transactions American Society of Civil Engineers, vol. 116, pp. 770-808, 1951.

[7] C.-K. Peng, S. V. Buldyrev, S. Havlin, M. Simons, H. E. Stanley, and A. L. Goldberger, "Mosaic organization of DNA nucleotides," Physical Review E, vol. 49, no. 2, pp. 1685-1689, 1994. 
[8] A. Castro E Silva and J. G. Moreira, "Roughness exponents to calculate multi-affine fractal exponents," Physica A: Statistical Mechanics and Its Applications, vol. 235, no. 3-4, pp. 327-333, 1997.

[9] R. O. Weber and P. Talkner, "Spectra and correlations of climate data from days to decades," Journal of Geophysical Research D: Atmospheres, vol. 106, no. 17, pp. 20131-20144, 2001.

[10] J. W. Kantelhardt, S. A. Zschiegner, E. Koscielny-Bunde, S. Havlin, A. Bunde, and H. E. Stanley, "Multifractal detrended fluctuation analysis of nonstationary time series," Physica A: Statistical Mechanics and Its Applications, vol. 316, no. 1-4, pp. 87-114, 2002.

[11] L. Zunino, A. Figliola, B. M. Tabak, D. G. Pérez, M. Garavaglia, and O. A. Rosso, "Multifractal structure in Latin-American market indices," Chaos, Solitons and Fractals, vol. 41, no. 5, pp. 2331-2340, 2009.

[12] B. Podobnik and H. E. Stanley, "Detrended cross-correlation analysis: a new method for analyzing two nonstationary time series," Physical Review Letters, vol. 100, no. 8, Article ID 084102, 2008.

[13] W.-X. Zhou, "Multifractal detrended cross-correlation analysis for two nonstationary signals," Physical Review E-Statistical, Nonlinear, and Soft Matter Physics, vol. 77, no. 6, Article ID 066211, 2008.

[14] Z.-Q. Jiang and W.-X. Zhou, "Multifractal detrending movingaverage cross-correlation analysis," Physical Review EStatistical, Nonlinear, and Soft Matter Physics, vol. 84, no. 1, Article ID 016106, 2011.

[15] G.-F. Gu and W.-X. Zhou, "Detrending moving average algorithm for multifractals," Physical Review E-Statistical, Nonlinear, and Soft Matter Physics, vol. 82, no. 1, Article ID 011136, 2010.

[16] E. Alessio, A. Carbone, G. Castelli, and V. Frappietro, "Secondorder moving average and scaling of stochastic time series," European Physical Journal B, vol. 27, no. 2, pp. 197-200, 2002.

[17] L. Kristoufek, "Multifractal height cross-correlation analysis: a new method for analyzing long-range cross-correlations," European Physics Letters, vol. 95, no. 6, Article ID 68001, 2011.

[18] W. Cheng and J. Wang, "Dependence phenomenon analysis of the stock market," European Physics Letters, vol. 102, no. 1, Article ID 18004, 2013.

[19] W. Fang and J. Wang, "Fluctuation behaviors of financial time series by a stochastic Ising system on a Sierpinski carpet lattice," Physica A: Statistical Mechanics and Its Applications, vol. 392, no. 18, pp. 4055-4063, 2013.

[20] W. Fang and J. Wang, "Statistical properties and multifractal behaviors of market returns by ising dynamic systems," International Journal of Modern Physics C, vol. 23, no. 3, Article ID 1250023, 2012.

[21] H. Niu and J. Wang, "Volatility clustering and long memory of financial time series and financial price model," Digital Signal Processing, vol. 23, no. 2, pp. 489-498, 2013.

[22] H. Niu and J. Wang, "Power-law scaling behavior analysis of financial time series model by voter interacting dynamic system," Journal of Applied Statistics, vol. 40, no. 10, 2013.

[23] J. Wang and S. Deng, "Fluctuations of interface statistical physics models applied to a stock market model," Nonlinear Analysis: Real World Applications, vol. 9, no. 2, pp. 718-723, 2008.

[24] J. Wang, Q. Wang, and J. Shao, "Fluctuations of stock price model by statistical physics systems," Mathematical and Computer Modelling, vol. 51, no. 5-6, pp. 431-440, 2010.
[25] T. Wang, J. Wang, J. Zhang, and W. Fang, "Voter interacting systems applied to Chinese stock markets," Mathematics and Computers in Simulation, vol. 81, no. 11, pp. 2492-2506, 2011.

[26] J. Zhang and J. Wang, "Fractal detrended fluctuation analysis of chinese energy markets," International Journal of Bifurcation and Chaos, vol. 20, no. 11, pp. 3753-3768, 2010.

[27] A. Pei and J. Wang, "Nonlinear analysis of return time series model by oriented percolation dynamic system," Abstract and Applied Analysis, vol. 2013, Article ID 612738, 12 pages, 2013.

[28] W. Y. Cheng and J. Wang, "Nonlinear fluctuation behavior of financial time series model by statistical physics system," Abstract and Applied Analysis, vol. 2014, Article ID 806271, 12 pages, 2014.

[29] R. Durrett, Lecture Notes on Particle Systems and Percolation, Wadsworth \& Brooks, Pacific Grove, Calif, USA, 1988.

[30] T. M. Liggett, Stochastic Interacting Systems: Contact, Voter and Exclusion Processes, vol. 324, Springer, New York, NY, USA, 1999.

[31] Y.-H. Shao, G.-F. Gu, Z.-Q. Jiang, W.-X. Zhou, and D. Sornette, "Comparing the performance of FA, DFA and DMA using different synthetic long-range correlated time series," Scientific Reports, vol. 2, article 835, 2012.

[32] M.-F. Chen, From Markov Chains to Non-Equilibrium Particle Systems, World Scientific, Singapore, 2nd edition, 1992.

[33] G. Grimmett, Percolation, vol. 321, Springer, Berlin, Germany, 2nd edition, 1999.

[34] T. M. Liggett, Interacting Particle Systems, vol. 276, Springer, New York, NY, USA, 1985.

[35] D. Lamberton and B. Lapeyre, Introduction to Stochastic Calculus Applied to Finance, Chapman \& Hall/CRC Financial Mathematics Series, CRC Press, London, UK, 1996.

[36] E. Bouyé, V. Durrleman, A. Nikeghbali et al., Copula for Finance: A Reading Guide and Applications, Financial Econometrics Research Centre, City University Business School, London, UK, 2000.

[37] U. Cherubini, E. Luciano, and W. Vecchiato, Copula Methods in Finance, Wiley Finance Series, John Wiley \& Sons, Chichester, UK, 2004.

[38] E. L. Lehmann, "Some concepts of dependence," Annals of Mathematical Statistics, vol. 37, pp. 1137-1153, 1966.

[39] S. Coles, J. Currie, and J. Tawn, Dependence Measures for Extreme Value Analysis, Department of Mathematics and Statistics, Lancaster University, 1999.

[40] M. Hollander and D. A. Wolfe, Nonparametric Statistical Methods, John Wiley \& Sons, New York, NY, USA, 1973.

[41] H. Joe, Multivariate Models and Dependence Concepts, vol. 73, Chapman \& Hall, London, UK, 1997.

[42] D. J. Blythe, "A rigorous and efficient asymptotic test for power-law cross-correlation,” Tech. Rep., 2013, http://arxiv-web3 .library.cornell.edu/abs/1309.4073.

[43] B. Podobnik, I. Grosse, D. Horvatić, S. Ilic, P. C. Ivanov, and H. E. Stanley, "Quantifying cross-correlations using local and global detrending approaches," European Physical Journal B, vol. 71, no. 2, pp. 243-250, 2009.

[44] E. Fama, "Efficient capital markets: a review of theory and empirical work," Journal of Finance, vol. 25, pp. 384-417, 1970.

[45] J. A. Busse and T. Clifton Green, "Market efficiency in real time," Journal of Financial Economics, vol. 65, no. 3, pp. 415-437, 2002.

[46] J. C. Reboredo, M. A. Rivera-Castro, J. G. V. Miranda, and R. García-Rubio, "How fast do stock prices adjust to market 
efficiency? Evidence from a detrended fluctuation analysis," Physica A, vol. 392, no. 7, pp. 1631-1637, 2013.

[47] X. Sun, H. Chen, Z. Wu, and Y. Yuan, "Multifractal analysis of Hang Seng index in Hong Kong stock market," Physica A, vol. 291, no. 1-4, pp. 553-562, 2001. 


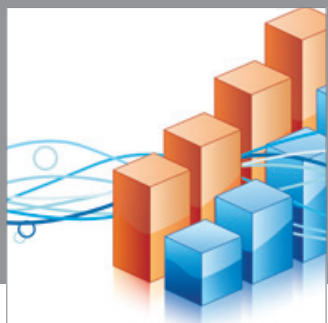

Advances in

Operations Research

mansans

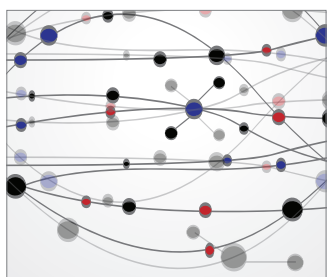

The Scientific World Journal
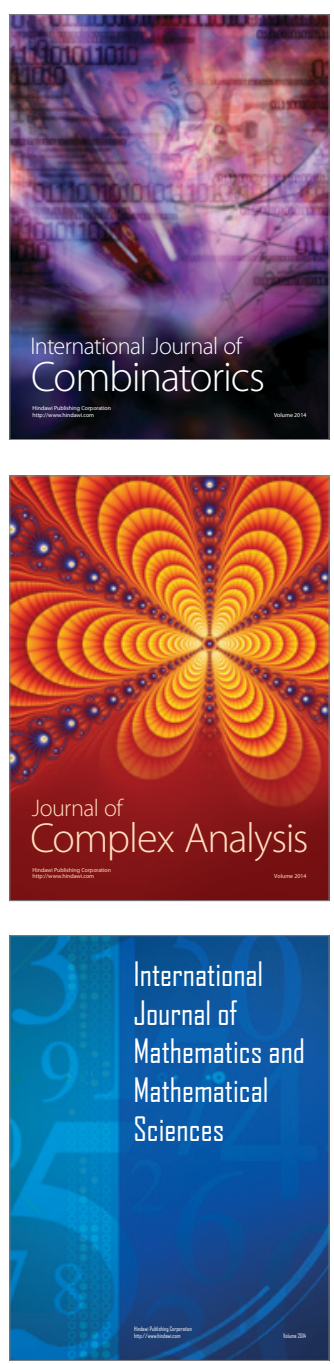
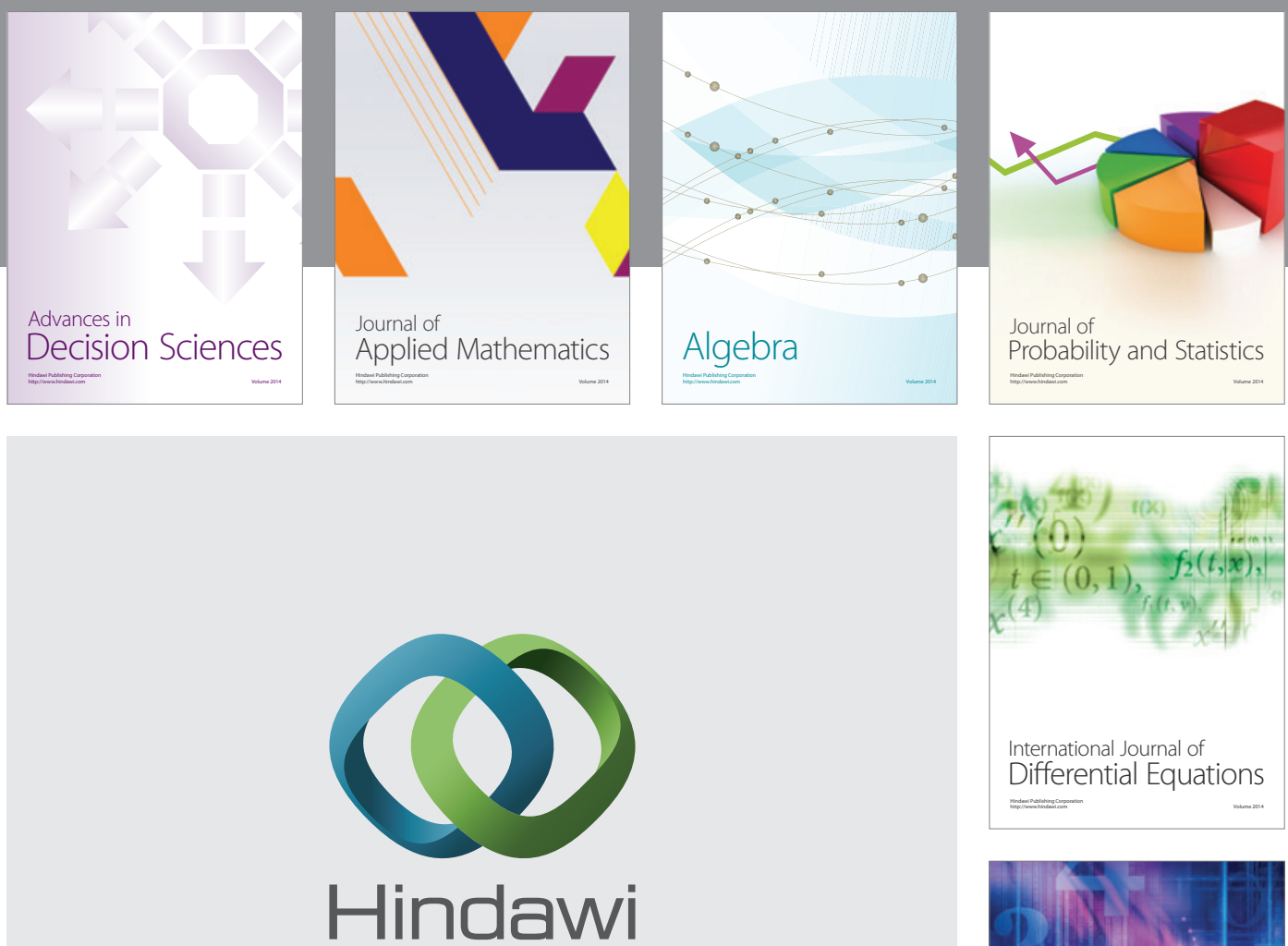

Submit your manuscripts at http://www.hindawi.com
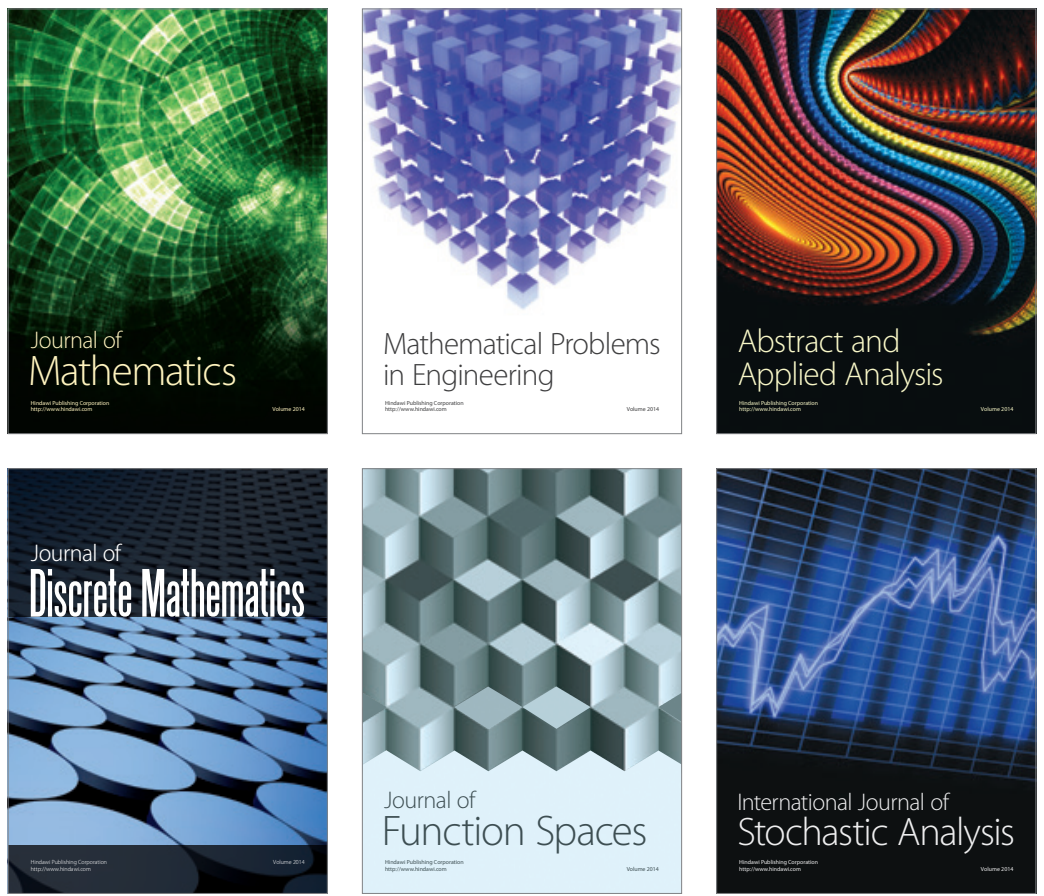

Journal of

Function Spaces

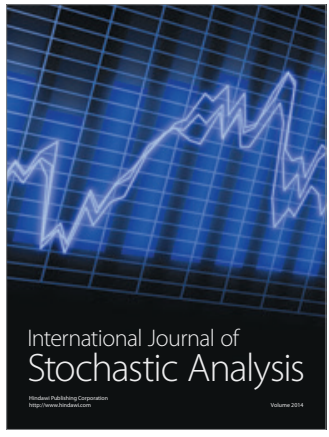

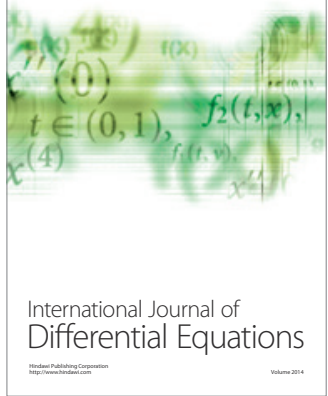
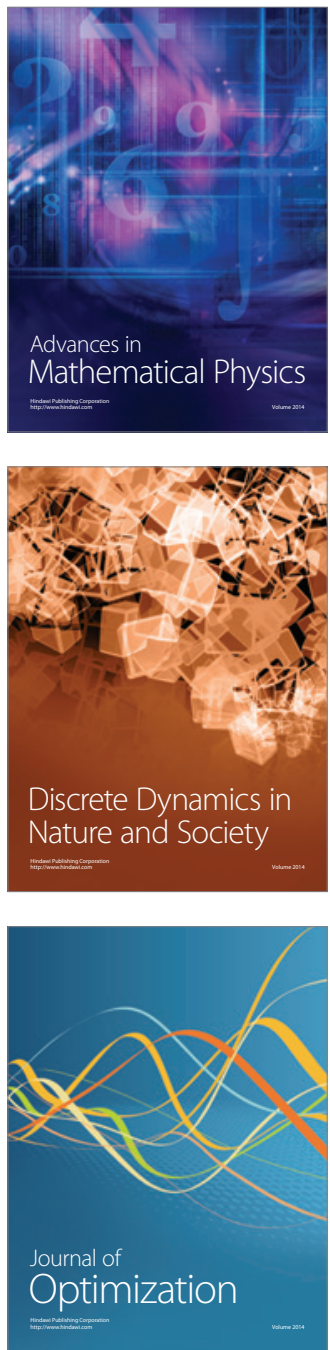